\title{
RECENSIÓN
}

\section{DESIGUALDADES SOCIALES EN SALUD: SITUACIÓN EN ESPAÑA EN LOS ÚLTIMOS AÑOS DEL SIGLO XX}

\author{
Enrique Regidor, Carme Borrell, M. Isabel Pasarín, Juan L. Guitiérrez-Fisac, Lourdes Lostao e \\ Iñaki Galán \\ Edita: Universidad de Alicante, 2002 \\ ISBN: 84-600-9727-7 \\ Núm. de páginas: 116 \\ Recensión por: Gloria Fernández-Mayoralas Fernández. Instituto de Economía y Geografía. Consejo Superior de \\ Investigaciones Científicas. \\ Correo electrónico: G.F.mayoralas@ieg.csic.es
}

Bajo el título Desigualdades sociales en salud: situación en España en los últimos años del siglo $X X$, libro coordinado por Enrique Regidor, encontramos cinco contribuciones heterogéneas, en objetivos y áreas de análisis, pero claramente comprometidas con la línea de investigación que estudia las desigualdades en salud como consecuencia de las circunstancias sociales y económicas de los individuos. Este enfoque tiene, como el mismo coordinador apunta, una importancia epistemológica enorme, ya que para comprender los mecanismos que generan las diferencias socioeconómicas en salud es preciso conocer adecuadamente los pasos o relaciones en la cadena de causalidad y no sólo controlar los efectos de unas y otras variables.

En la primera de estas contribuciones, Enrique Regidor realiza una revisión teórica de los determinantes socioeconómicos de la salud, destacando la escasa aportación de los factores de riesgo individuales en la explicación de las desigualdades en salud, en tanto en cuanto persiste el gradiente social aunque éstos sean controlados. Más aún, imputa la importancia atribuida a estos factores a la creencia implícita de que es más difícil modificar el medio ambiente que cambiar las conductas de los individuos, a pesar de que no siempre intervenciones in- tensivas con este fin llevan asociadas cambios conductuales notables. De manera que los mayores determinantes de la salud de la población serían lo que él denomina factores del riesgo económico y social, que reflejan la situación social y económica de los individuos, como la clase social basada en la ocupación, la educación o nivel de estudios, los ingresos económicos, el grado de privación material o el área de residencia. Finalmente, revisa los mecanismos que han sido propuestos para explicar la relación entre medio y salud: utilización de servicios sanitarios, procesos de selección, recursos económicos y sociales que modifican la susceptibilidad a la enfermedad, exposición a factores de riesgo biológico y de conducta y su gradiente social, circunstancias materiales, factores psicosociales y antecedentes familiares en lo social y económico.

La segunda de las contribuciones, por Carme Borrell y M Isabel Pasarín, se centra en la influencia de las desigualdades sociales sobre la evolución de la mortalidad durante la última década del siglo xx en la ciudad de Barcelona. Tras esbozar algunos aspectos sociodemográficos de la ciudad y hacer una revisión de estudios anteriores sobre diferencias en mortalidad, las autoras comparan las tasas de mortalidad y mortalidad prematura (años potenciales de vida 
perdidos de 1 a 70 años), ambas estandarizadas por edad, de dos agrupaciones de barrios según su nivel socioeconómico, describiendo los resultados por sexo, tanto de la debida a todas las causas como distinguiendo por tipo de enfermedad (infecciosas, cáncer, cardiovasculares, respiratorias, del aparato digestivo y causas externas). No obstante, echamos en falta un mapa o un anexo que explicite qué barrios conforman cada una de las agrupaciones y bajo qué criterios se incluyen los barrios en una u otra agrupación. Aunque para este análisis no han sido utilizadas las variables del Padrón Municipal de Habitantes, el registro de mortalidad de la ciudad de Barcelona tiene la ventaja, deseable para ser imitada en otras áreas del estado español, de estar conectado con esta fuente de información básica en lo demográfico y socioeconómico.

Precisamente la ausencia de registros que enlacen información socioeconómica y de salud es lo que impide, en muchas ocasiones, desarrollar estudios sobre desigualdades sociales en salud. Una fuente que permite soslayar parte de este obstáculo es la encuesta de salud al cuestionar asimismo sobre la posición socioeconómica de los individuos. En España se han realizado encuestas de salud periódicamente desde 1987 hasta 1997, y manteniendo la mayoría de sus directrices metodológicas, lo que posibilita además llevar a cabo análisis de la evolución de las desigualdades sociales en aspectos relevantes, como es el caso de la salud percibida o los trastornos crónicos declarados. Este ha sido el objetivo de la contribución de Juan Luis Gutiérrez-Fisac, que analiza la evolución de la autopercepción de la salud así como de cinco transtornos crónicos, según el nivel de estudios y el sexo de la población española, entre 1987 y 1995/97, concluyendo que se ha producido un incremento en las diferencias socioeconómicas en la salud a pesar de acontecer, como el mismo autor reconoce, una mejora sustancial en los principales indicadores de salud y de un aumento en el nivel socioeconómico de la población en su conjunto. La- mentablemente, sólo disponemos de dos puntos de corte para poder evaluar la tendencia futura, por lo que hubiera sido interesante incluir en el estudio los datos de la Encuesta de Salud de 1993.

También las Encuestas de Salud españolas proveen de información sobre utilización y accesibilidad a los servicios sanitarios, permitiendo estudiar hasta qué punto las desigualdades sociales en salud pueden minimizarse en países que, como el nuestro, disfrutan de sistemas de cobertura universal. De este asunto se ocupa Lourdes Lostao en su contribución sobre diferencias en la utilización y accesibilidad a los servicios sanitarios en España, de acuerdo al nivel socioeconómico y el nivel de estudios de la población usuaria. La autora señala que entre 1987 y 1995/97 se ha producido un decremento de las diferencias en la utilización de la consulta médica, pero persistiendo las diferencias en el tiempo de espera para consulta; se ha mantenido la ausencia de diferencias en el ingreso hospitalario desapareciendo, además, las diferencias en el tiempo de espera para ingreso; sin embargo continúan las diferencias socioeconómicas en la utilización de servicios preventivos. Y, todo ello, en línea con los resultados observados en otros países de nuestro entorno cultural lo que, en todo caso y en palabras de la autora no ha reducido la desigualdad en términos de salud entre las clases sociales.

La quinta y última contribución del libro, a cargo de Iñaki Galán, centra su atención en las desigualdades sociales en los hábitos de consumo de tabaco y alcohol, actividad física y dieta alimenticia, que tiene la población de la Comunidad de Madrid, utilizando los datos del Sistema de Vigilancia de Factores de Riesgo asociados a Enfermedades no Transmisibles, que se recogen mensualmente mediante entrevista telefónica a una muestra de población adulta de entre 18 y 64 años. Teniendo en cuenta esta información, el autor analiza la evolución entre 1995-97 y 1998-2000 de una serie de parámetros (fu- 
madores diarios, abandono de tabaquismo, consumidores de alcohol de riesgo, individuos con test de CAGE positivo, inactividad física en tiempo libre, consumo de fruta y verdura menos de tres veces/día), según el nivel de estudios y la clase social de los entrevistados, y compara sus resultados con los obtenidos en el Eurobarómetro así como por encuestas en otras áreas del mismo contexto socioeconómico, dentro y fuera del estado español.

En definitiva, y a pesar de que la asociación entre desigualdad socioeconómica $\mathrm{y}$ salud pueda ser puesta en duda en países occidentales industrializados (BMJ, 2002, 324:1-2), la exigencia de rigor en la selección de áreas, calidad de los datos, uso de controles y descarte de factores de confusión, no debe ser motivo de preocupación para los investigadores en esta disciplina, ya que la exposición de hipótesis y su refutación es la propia finalidad de la ciencia. En este sentido, confiamos que estudios como los que componen este libro se normalicen y proliferen, consiguiendo impregnar las políticas sociales y económicas en materia de salud. 